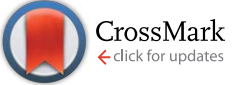

Cite this: RSC Adv., 2016, 6, 40489

\title{
Effects of water and alcohols on the polymerization of furan during its acid-catalyzed conversion into benzofuran $\dagger$
}

\author{
Xun Hu, Shengjuan Jiang, Sri Kadarwati, Dehua Dong and Chun-Zhu Li* \\ Furan, an important product from catalytic pyrolysis of biomass, has the potential to be further converted \\ into value-added chemicals or biofuels. This study investigated the conversion of furan into benzofuran \\ over a Brønsted acid catalyst (Amberlyst 70) at $140-190{ }^{\circ} \mathrm{C}$ in various solvents. With water as the solvent, \\ furan could barely make its way to benzofuran as its polymerization dominated. With methanol as the \\ solvent, the polymerization of furan was suppressed and benzofuran formation was enhanced \\ substantially. This is because in methanol, the reactive intermediates (i.e., aldehydes) were stabilized and \\ their involvement in polymerization reactions was suppressed. Other alcohols showed similar effects on \\ suppressing polymerization. In dimethyl sulfoxide (DMSO), the polymerization of furan was also \\ effectively suppressed. However, furan was not converted to benzofuran but to levulinic acid via \\ a distinct reaction route.
}

Received 23rd February 2016 Accepted 5th April 2016

DOI: $10.1039 / c 6 r a 04745 a$

www.rsc.org/advances

\section{Introduction}

Bio-oil, a crude liquid produced from pyrolysis of biomass, is a sustainable feedstock for the production of biofuels and fine chemicals. However, bio-oil tends to be polymerized, especially at elevated temperatures, ${ }^{1}$ which leads to the deactivation of catalyst $^{2}$ or blockage of the reactor. ${ }^{3}$ Understanding how the main components of bio-oil (i.e. furans, sugars and phenolics) contribute to the polymerization reactions is essential to develop the methods for the stabilization of bio-oil.

The furans in bio-oil present in forms such as 5-(hydroxymethyl)furfural (HMF), furfural, 5-methyl-2-furancarboxaldehyde, acetylfuran and furfuryl alcohol. ${ }^{4}$ The functionalities of these furans are different though they all have the furan ring. Thus, we started our investigation from the simplest of furans-furan itself-to understand how furan polymerizes and how to stabilize it. Furan is an important product in the catalytic pyrolysis of biomass..$^{5-7}$ However, unlike furfural ${ }^{8-12}$ or 5(hydroxymethyl)furfural (HMF), ${ }^{13-16}$ the catalytic conversion of furan (i.e. via acid catalysis) has received much less attention.

Esterification is an important method for upgrading bio-oil by transforming the carboxylic acids and other reactive components. This is generally performed in the presence of an acid catalyst at elevated reaction temperatures $\left(c a .170{ }^{\circ} \mathrm{C}\right) .{ }^{17} \mathrm{In}$ this study, we initially intended to investigate the

Fuels and Energy Technology Institute, Curtin University of Technology, GPO Box U1987, Perth, WA 6845, Australia. E-mail: chun-zhu.li@curtin.edu.au; Fax: +618 9266 1131; Tel: +61892661138

$\dagger$ Electronic supplementary information (ESI) available. See DOI: 10.1039/c6ra04745a polymerization tendency of furan under the conditions of biooil esterification. However, the unexpected conversion of furan into benzofuran was observed. It has been reported that furan could be converted into benzofuran, an intermediate to make aromatic range biofuels, but via gas-phase reactions over ZSM-5 or HZSM-5 at $300-700{ }^{\circ} \mathrm{C} . .^{18-21}$

In this study, we have developed a new approach for the conversion of furan into benzofuran. In the liquid medium (methanol as the solvent/reactant), with the aid of a Brønsted acid (Amberlyst 70), furan was converted into benzofuran at a temperature as low as $150{ }^{\circ} \mathrm{C}$. To the best of our knowledge, this is the first time the conversion of furan into benzofuran has been reported in a liquid phase over a Brønsted acid at such a low reaction temperature. In addition, we found that solvents significantly affect the reaction network of furan. For example, in dimethyl sulfoxide (DMSO), furan was converted into levulinic acid, not benzofuran, representing a new route for the production of levulinic acid from furan.

In the initial trials for the conversion of furan into benzofuran, we found that, in water, furan had an extremely high tendency towards polymerization with almost no chance to form benzofuran. Our method to prevent the polymerization used alcohols to stabilize the reactive aldehyde intermediates by converting them into acetals. In this way, the polymerization of furan could be suppressed to a significant extent, while the formation of benzofuran was effectively enhanced.

In our previous studies, ${ }^{13,22-24}$ we found that the polymerization of sugars and HMF could be effectively suppressed in methanol medium. In this study, we found that the polymerization of furan could also be suppressed in methanol. However, there are several major differences in terms of the type of 
reactions and intermediates involved in the conversion of sugars and the conversion of furan. First, the conversion of the C6 sugars into levulinic acid involves the dehydration of sugars to form HMF and the following decomposition of HMF to form levulinic and formic acids. ${ }^{\mathbf{1 3 2 2}}$ In comparison, the conversion of furan into benzofuran involves the opening of the furan ring and electrophilic substitution reactions. We found that methanol could suppress polymerization during the opening of the furan ring and the electrophilic substitutions reactions. This is different from the suppression of polymerization reactions during the dehydration of sugars and the decomposition of HMF. Second, comparing with HMF, furan does not have either the hydroxyl group or the carbonyl group, while methanol as the reactant/solvent could still suppress the polymerization of furan during the opening of the furan ring. This is new when compared with the previous studies..$^{\mathbf{1 3 2 2 - 2 4}}$ Third, in this study we found that methanol could stabilize and retain the soluble polymers in the reaction medium, slowing down the further conversion of soluble polymer into insoluble polymer. This constitutes new knowledge about the mechanism for the suppression of polymerization reactions in methanol.

\section{Experimental}

\section{Materials}

The chemicals used in this study were obtained commercially and were of analytical grade. Amberlyst 70 is a commercially available solid acidic resin catalyst (Dow Chemical). The acid density is $>2.55 \mathrm{mmol} \mathrm{g}^{-1}$ and the maximum operating temperature is $190{ }^{\circ} \mathrm{C}$. The catalyst was used directly without any pretreatment. This study involves the use of furan and benzofuran. Researchers need to pay particular attention in handling these chemicals due to their potential health hazards. Furan is an extremely flammable liquid with a low boiling point $\left(31.3^{\circ} \mathrm{C}\right)$. It can cause skin irritation and damage to organs through prolonged or repeated exposure. It also may cause cancer and is suspected of causing genetic defects. Personal protective equipment, such as lab coat, gloves, and masks, are required for dealing with this chemical. The operations with furan have to be undertaken in a fume cupboard or in a well ventilated area. Benzofuran is flammable and is also suspected of causing cancer. Personal protective equipment and a well ventilated area are also required for dealing with benzofuran.

\section{Experimental procedure}

The acid-catalyzed conversion of furan was performed in a Hastelloy autoclave reactor. The experimental procedure was similar to that in our previous studies. ${ }^{22,25}$ In brief, furan, solvent(s) and Amberlyst 70 were mixed and loaded into the reactor at room temperature. The reactor was assembled and the residual air inside was replaced with nitrogen by purging the reactor. The detailed purging procedure was as follows.

The reactor was pressurized to 30 bar with nitrogen at room temperature. Subsequently, the pressure was released slowly to ca. 1 or 2 bar and the released valve was then turned off immediately. The same procedure was repeated three times. It should be noted that furan is very volatile and is not dissolved in water. During the purging process, some furan might be carried away with the nitrogen. To confirm this, blank experiments were performed to measure the loss of furan with water and methanol as the solvents during the purging process. The results showed that in water after the pressurization and depressurization processes, around $17 \mathrm{wt} \%$ of furan was lost. The loss of furan in methanol is negligible $(<1 \mathrm{wt} \%)$. The data in the figures/tables has been calibrated by considering the loss of furan during the purging process.

After the purging with nitrogen, the reactor was heated to the required reaction temperatures $\left(120-190{ }^{\circ} \mathrm{C}\right)$ over 10 to $14 \mathrm{~min}$ with a stirring rate of $350 \mathrm{rpm}$ under the vapour pressure generated from the reactants. For some experiments, sampling was performed at a certain time interval to understand the progress of the reactions. A sampling tube with one outlet extending to the bottom of the reactor vessel was used to take liquid samples. A stirrer was used to stir the reactant mixture. When water was used as the solvent, the samples taken from the reactor were first weighed and then mixed with THF of a similar volume and weighed again. Such sampling procedure was not applied when methanol was used as the solvent as methanol can effectively dissolve furan. For the experiments aimed to measure the formation of insoluble polymer, no sampling was performed. After cooling the reactor by a cooling coil inside the reactor vessel, the liquid and the solid were collected and separated by filtering. The solid residue was then dried at $105^{\circ} \mathrm{C}$ for $c a .4$ hours to measure the amount of the insoluble polymer formed. Because the solid residue included the catalyst, the weight of the catalyst (on a moisture-free basis) was subtracted to obtain the weight of the insoluble polymer (on a moisturefree basis).

\section{Analytical methods}

The liquid samples were analyzed with an Agilent GC/MS (6890 series GC with a 5973 MS detector) equipped with a HPINNOWax capillary column. The analysis procedures are detailed eleswhere. ${ }^{26}$ Synchronous spectra of the soluble polymers in the liquid samples were acquired with a PerkinElmer LS50B spectrometer. The energy difference used was $-2800 \mathrm{~cm}^{-1}$ and the scan speed was $200 \mathrm{~nm} \mathrm{~min}^{-1}$. The UV fluorescence was mainly used to characterize the $\pi$-conjugated structures in the soluble polymers. The $\pi$-conjugated structures can be a single or a fused aromatic ring system or a longchain alkene with $\pi$-conjugation. The emission spectrum is related to the size of the $\pi$-conjugated structures and also their abundance in the solution. In general, the bigger the $\pi$ conjugated structure, the longer the wavelength of the spectrum.

The polymers formed inside the catalyst were also characterized with a Perkin-Elmer Spectrum GX FT-IR/Raman spectrometer to probe their functionalities. Because the polymer cannot be separated from the catalyst, the polymer and catalyst were thus analyzed together. The fresh catalyst was also 
analyzed for comparison. The spectrometer has a spectral resolution of $4 \mathrm{~cm}^{-1}$. The samples were milled together with $\mathrm{KBr}$ with a concentration of $1 \mathrm{wt} \%$. The pressed pellet was scanned 20 times and the spectrum represents the average.

Elemental composition of the polymer plus catalyst was determined with an elemental analyzer. Because it is almost impossible to separate the polymer from the catalyst, they were analyzed together. The fresh catalyst was also analyzed with the element analyzer and the $\mathrm{C}, \mathrm{H}, \mathrm{S}$, and $\mathrm{O}$ contents in the fresh catalyst were obtained directly. The elemental contents of the polymer could not be obtained directly as from the element analyzer, only the total $\mathrm{C}, \mathrm{H}, \mathrm{S}$, and $\mathrm{O}$ contents were obtained. However, because the amount of polymer on/in the catalyst is known, the elemental composition of the polymer can then be calculated based on the mass balance. For example, the carbon content of the polymer can be calculated by the following mass balance equation:

$$
\begin{aligned}
c_{\text {polymer }} \times m_{\text {polymer }}+c_{\text {catalyst }} \times m_{\text {polymer }}= & m_{\text {catalyst and polymer }} \\
& \times c_{\text {total }}
\end{aligned}
$$

where $c$ is the carbon content. Hydrogen content was also calculated in a similar way. The content of oxygen was calculated by difference. The element content was based on a dry ashfree basis.

Calculation of the carbon mass balance was based on the results of the elemental analysis. The carbon balance for the acid-catalyzed conversion of furan in water was typically $>95 \%$.

The yield of the insoluble polymer (\%, on a dry weight basis) was calculated by the equation:

Yield $(\%)=$

(weight of the solid collected - weight of the fresh catalyst) weight of furan loaded

$\times 100$

The yield of benzofuran (molar basis) was defined by the equation:

$$
\text { Yield }(\operatorname{mol} \%)=\frac{\text { moles of benzofuran produced } \times 2}{\text { moles of furan loaded }} \times 100
$$

The selectivity of benzofuran was defined by the following equation:

$$
\text { Selectivity }(\%)=\frac{\text { moles of benzofuran produced } \times 2}{\text { moles of furan converted }} \times 100
$$

It should be noted herein that formation of 1 mol benzofuran consumes $2 \mathrm{~mol}$ of furan and therefore the amount of benzofuran produced (molar basis) has to be multiplied by 2 to calculate the yield.

The yield of levulinic acid was defined by the equation:

$$
\text { Yield }\left(\mathrm{mol}^{\%}\right)=\frac{\text { moles of levulinic acid produced }}{\text { moles of furan loaded }} \times 100
$$

\section{Results and discussion}

\section{Conversion of furan into polymer in water}

Water is commonly used as a medium for the hydrolysis of biomass, sugars and furans. ${ }^{27-29}$ Therefore, we initially tried to convert furan in water over Amberlyst 70 (A70) (Fig. 1). Although furan was converted completely (Fig. 1a), only a small amount of benzofuran was produced (Fig. 1b). Succinaldehyde was another detectable product with a negligible concentration (Fig. S1 $\dagger$ ). The mass balance based on the detectable products was extremely low. Therefore, we suspected that a significant amount of polymer was formed. The following experiment confirmed our hypothesis (Fig. 2). The yield of the insoluble polymer from the conversion of furan in water was around $90 \%$ (weight basis) and benzofuran was produced in a negligible level $(<1 \%)$. Clearly, the polymerization was preferred and dominated the reaction pathway for the acid-catalyzed conversion of furan in water.

In water, the furan ring can be opened to form succinaldehyde (Scheme 1) under the acidic conditions. The carbonyl group of aldehydes has been known to play essential roles in polymerization reactions. ${ }^{\mathbf{3 0}, 31}$ Succinaldehyde has carbonyl groups and two active $\alpha$-H's, making it a very reactive electrophile in the Aldol condensation and in electrophilic substitution/addition reactions. The electron density of the furan ring is even higher than that of the benzene ring due to conjugation of the isolated electron pair on oxygen (in the furan ring) with the carbon-carbon double bonds, which makes it a good target for electrophiles such as succinaldehyde.

It was likely that furan polymerized with succinaldehyde, decreasing its further conversion into benzofuran. Succinaldehyde was possibly one key intermediate inducing the polymerization reactions. The high reactivity of succinaldehyde towards polymerization originates from its carbonyl groups. If the carbonyl groups were transformed into acetals via reaction with methanol, the involvement of succinaldehyde in the polymerization might be suppressed. This concept was confirmed by the following experiments with methanol as the solvent.

\section{Conversion of furan into benzofuran in methanol}

In methanol, benzofuran was formed even at temperatures as low as $150{ }^{\circ} \mathrm{C}$ (Fig. 1b). Increasing the reaction temperature to $170{ }^{\circ} \mathrm{C}$ further promoted its formation. Succinaldehyde, the intermediate detected in water, was not detected in methanol. Instead, 1,1,4,4-tetramethoxybutane, the acetal of succinaldehyde, was formed as the main product (Fig. S2 $\dagger$ and Scheme 1). 1,1,4,4-Tetramethoxybutane could maintain its presence in the acidic medium for a prolonged reaction time (Fig. 1c), indicating its improved stability. This was the important reason for the suppression of the polymerization reactions in methanol.

The identification of 1,1,4,4-tetramethoxybutane was the key to understand the roles of methanol in the suppression of the polymerization reactions. Its mass spectrum and the proposed dissociation routes in the mass spectrometer are shown in Fig. S3 and S4, $\dagger$ respectively. Further to this, methanol also reacted with furan via electrophilic addition to form 

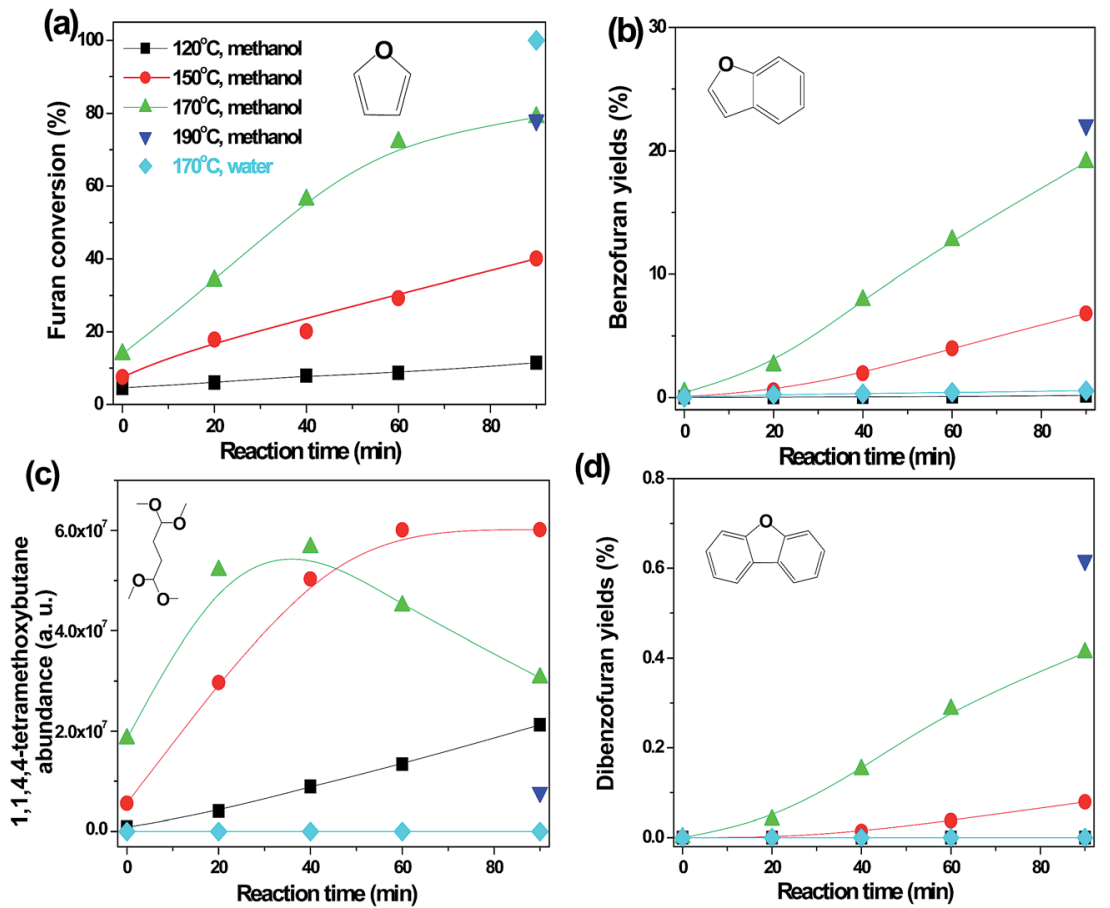

Fig. 1 The conversion of furan in methanol and water. Reaction conditions: furan, 3 g; solvent, 100 ml; catalyst, A70, 3 g; stirring rate, 350 rpm; reaction time, $90 \mathrm{~min}$; pressure, autogenous vapor pressure. (a) The conversion of furan. The conversion of furan in water was measured by mixing the residual products with THF as furan does not dissolve in water. (b) The yields of benzofuran; (c) the abundance of 1,1,4,4-tetramethoxybutane; (d) the yields of dibenzofuran. In the experiment at $190^{\circ} \mathrm{C}$ in methanol, no sampling was performed. " 0 min" in the $x$-axis means the reaction temperature just reached the required temperature.
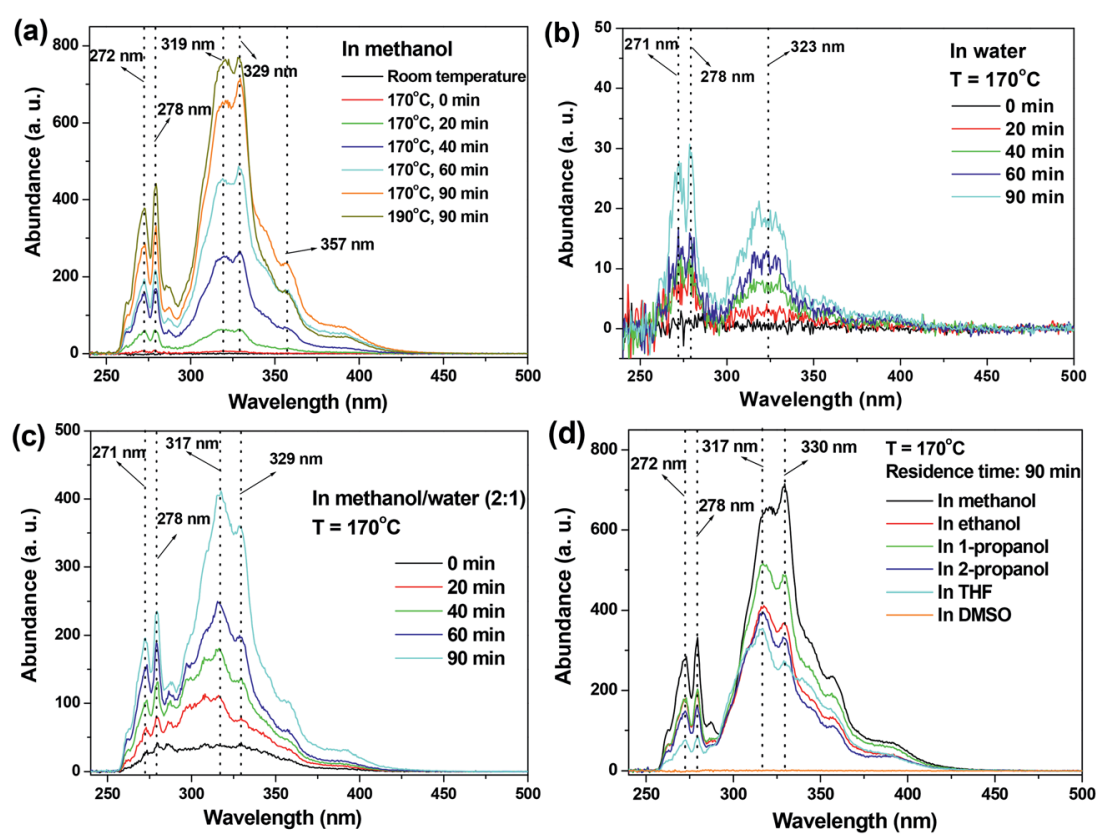

Fig. 2 Constant energy $\left(-2800 \mathrm{~cm}^{-1}\right.$ ) synchronous spectra for the soluble polymers from the acid-treatment of furan in the various solvents. (a) Spectra of the soluble polymers versus the reaction time in methanol; (b) spectra of the soluble polymers versus the reaction time in water; (c) spectra of the soluble polymers versus the reaction time in methanol/water; (d) spectra of the soluble polymers versus solvents. The concentration of the samples prepared for UV fluorescence analysis was 1600 ppm in Fig. 3c, and others were 400 ppm. The fluorescence peaks at ca. 271 and $278 \mathrm{~nm}$ are attributed to benzofuran and dibenzofuran, respectively. The fluorescence peaks at ca. 317 to $329 \mathrm{~nm}$ are roughly attributed to the compounds with three fused benzene rings. The fluorescence peaks above $360 \mathrm{~nm}$ are roughly attributed to the compounds with four or more fused benzene rings. 


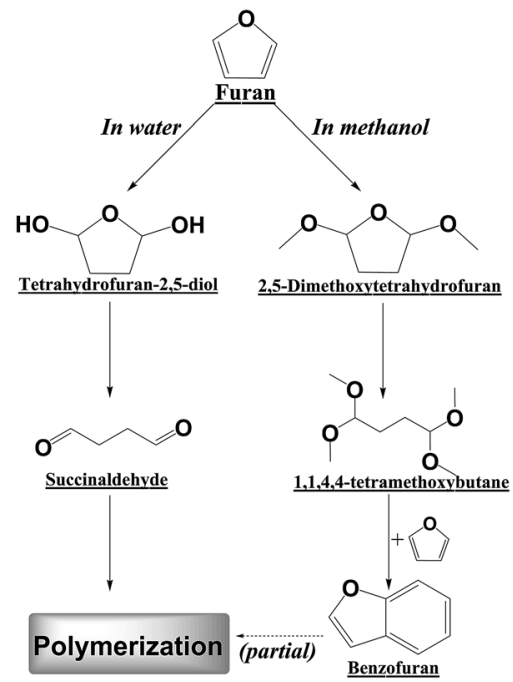

Scheme 1 The distribution of the main products during the conversion of furan in water and in methanol. All the compounds listed above except the polymer were detected with GC-MS. Tetrahydrofuran-2,5diol, succinaldehyde, 2,5-dimethoxytetrahydrofuran and 1,1,4,4-tetramethoxybutane are the intermediates. The opening of the furan ring, the acetalization, the formation of benzofuran and the polymer were believed to be the acid-catalyzed reactions.

2,5-dimethoxytetrahydrofuran with the cis-/trans-isomers (Scheme 2), which apparently proceeded in a stepwise manner. The formation of 2,5-dimethoxytetrahydrofuran eliminated the carbon-carbon double bonds in the furan ring and minimized the involvement of furan in the polymerization.

Benzofuran was the main product in methanol and its formation was promoted at elevated temperatures (Fig. 1b). However, benzofuran was not stable in the acidic environment, as the "furan ring" was still preserved in benzofuran. It continued to condense with furan to form dibenzofuran (Fig. 1d). The further increase of reaction temperature from 170 to $190{ }^{\circ} \mathrm{C}$ did not yield significantly more benzofuran (Fig. 1b) but much more dibenzofuran (Fig. 1d). In fact, the formation of dibenzofuran occurred almost simultaneously with the formation of benzofuran (Fig. $1 \mathrm{~b}$ and $\mathrm{d}$ ).

In water, the furan ring could be opened to form succinaldehyde. In methanol, the furan ring could also be opened to form 1,1,4,4-tetramethoxybutane. It is likely that the formation of benzofuran in methanol proceeded via the ring-opening route with 1,1,4,4-tetramethoxybutane as the intermediate product. The proposed reaction pathways involved three main steps, as depicted in Scheme 3. The initial step was the attack on $\mathrm{C} 2$ or $\mathrm{C} 5$ of the furan ring by a proton to form the carbon cation. Further attack on oxygen in the furan ring resulted in the opening of the furan ring and the formation of 1,1,4,4-tetramethoxybutane via the acetalization. The methoxy group in the acetal could then be removed via the formation of methanol (Scheme 3), producing the carbocation. The carbocation could react with the furan with its high electron density via electrophilic substitution. The further removal of the methoxy group followed by the subsequent electrophilic substitution eventually formed benzofuran. The electrophilic substitution reaction was the key step for the formation of the benzene ring in benzofuran, which was also reported as the main reaction pathway for benzofuran formation via the gas-phase condensation of furan..$^{20}$

Benzofuran continued to react with the intermediate derived from furan, forming dibenzofuran. The formation of benzofuran and dibenzofuran possibly involved similar intermediate(s) derived from the opening of the furan ring. Dibenzofuran, like benzofuran, was also not stable. It was further hydrolyzed to 2,6'-biphenyldiol (Scheme 2). The means to control specific selectivity in the acid-catalyzed conversion of furan is another challenge apart from the polymerization. Corma et al. developed a process for the production of alkanes $\left(\mathrm{C}_{14} \mathrm{H}_{30}\right.$ and higher, the diesel range fuel) via the acid-catalysed alkylations of 2-methylfuran and the following hydrogenation. ${ }^{32,33}$ In this

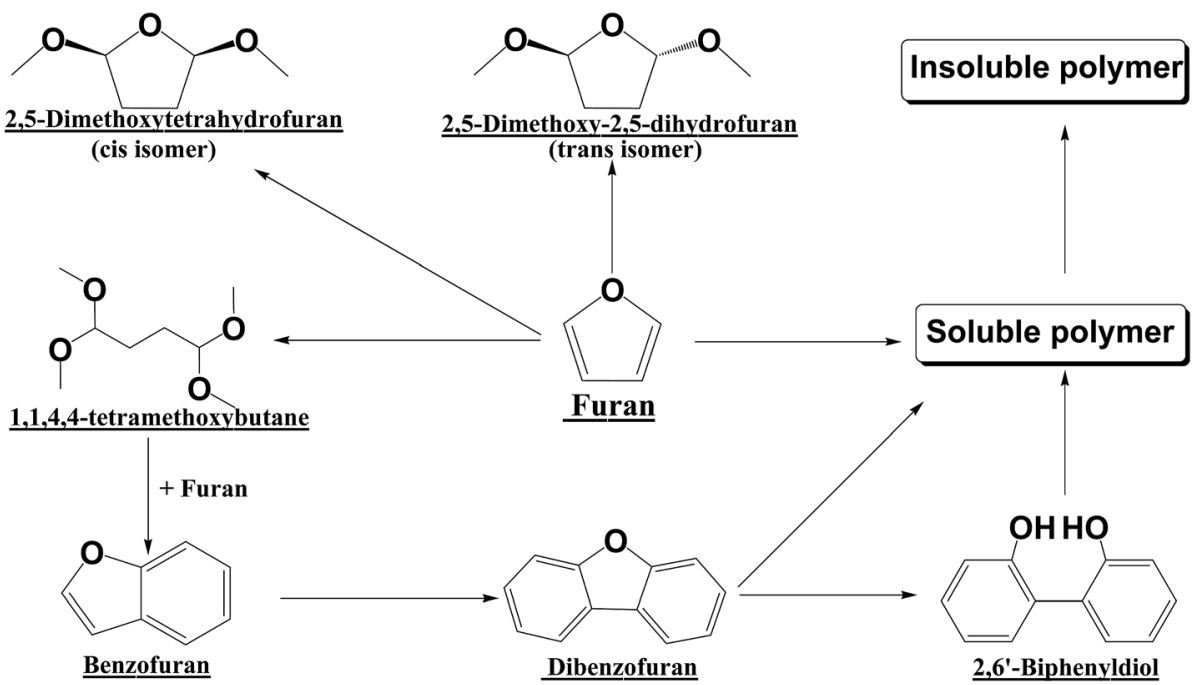

Scheme 2 A proposed reaction network for the conversion of furan in methanol. All the compounds listed above except the polymer were detected with GC-MS. 

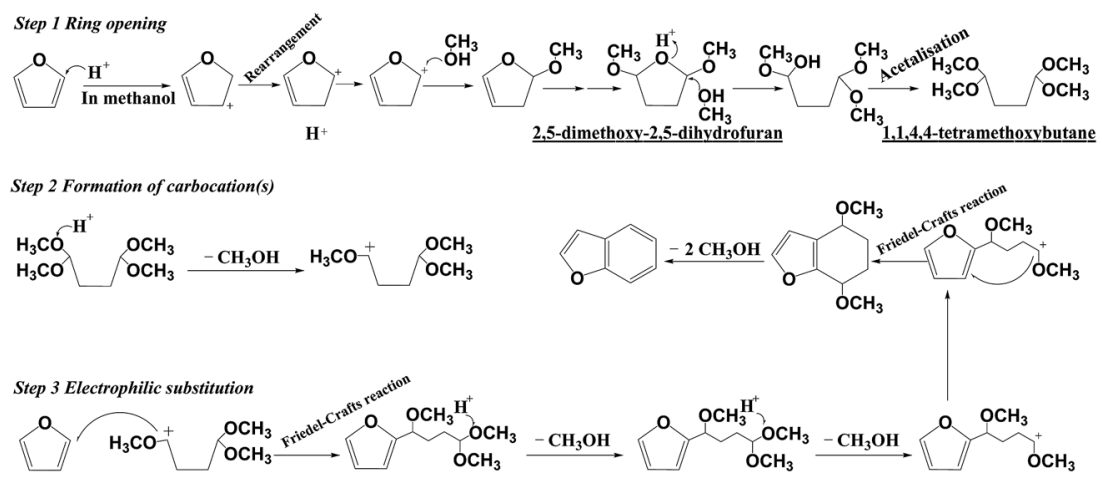

Scheme 3 Proposed reaction pathways for the formation of benzofuran from furan via the ring-opening route in methanol. The intermediate, 1,1,4,4-tetramethoxybutane, was detected with GC-MS.

study, benzofuran was produced from furan via two alkylation reactions in the presence of an acid catalyst. Without following a hydrogenation step, the rings in benzofuran were retained. It is believed that if hydrogen and a hydrogenation catalyst were also present in the reaction medium, C8 alkanes, instead of benzofuran, would be produced.

\section{Polymerization of furan in methanol}

The polymerization could be significantly suppressed in methanol, but not be eliminated completely, even at temperatures as low as $150^{\circ} \mathrm{C}$, as evidenced by the formation of the soluble polymers (Fig. S5†). This might be explained in three ways. First, benzofuran and dibenzofuran were still reactive. Benzofuran could multiply to form dibenzofuran, whereas dibenzofuran could hydrolyze to 2,6'-biphenyldiol, which could not be prevented by methanol. These products might continue their way to form bigger molecules such as soluble polymers (Fig. 2a).

Second, methanol could not stabilize all the compounds containing carbonyl groups. In addition to the $\pi$-conjugated structures in the soluble polymer (Fig. 2), aromatic ketone functionalities were also identified by the characterization of the soluble polymer with FT-IR (Fig. 3). The IR absorption at $1670-1650 \mathrm{~cm}^{-1}$ was assigned typically to aromatic ketones, ${ }^{34}$ but not to aliphatic aldehydes with a typical absorption at 1740$1720 \mathrm{~cm}^{-1}$. $^{34}$ The abundance of aromatic ketones was increased monotonically with the progress of the reaction (Fig. $3 \mathrm{~b}$ ). This result was in line with the monotonic increase in the abundance of the $\pi$-conjugated structures with the reaction time (Fig. 2a). The aromatic ketone groups were possibly assigned to the functionalities of the heavy products (i.e. soluble polymers) as the detectable light products did not contain the aromatic ketone functionality. Methanol could transform the aliphatic aldehydes such as succinaldehyde into acetals, but was not able to transform the aromatic ketones effectively. With the progress of the reaction, these compounds continue to polymerize.

Third, a significant amount of water was formed during the acid-catalyzed conversion of furan in methanol. In the presence of the acid catalyst, a part of the methanol underwent intermolecular etherification, forming dimethyl ether and water.
The concentration of water in the acid treatment of furan in methanol at $170{ }^{\circ} \mathrm{C}$ reached $c a .14 \mathrm{wt} \%$ at the end. The presence of water in methanol had significant effects on the formation of benzofuran, which was confirmed by the following experiments
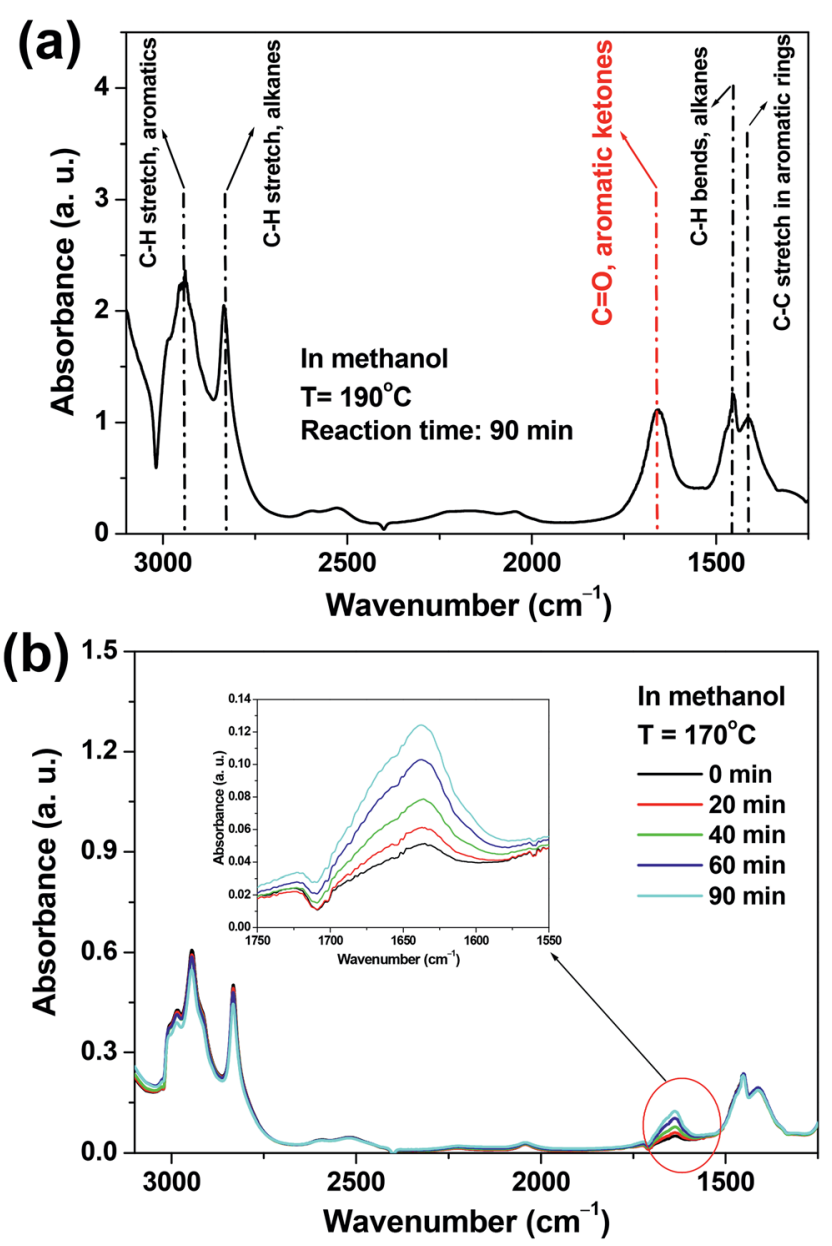

Fig. 3 The FT-IR characterization of the liquid samples from the acidtreatment of furan in methanol at (a) $T=190^{\circ} \mathrm{C}$ and (b) $T=170^{\circ} \mathrm{C}$. The sample in Fig. 4 a was analyzed directly without dilution. The samples in Fig. $4 \mathrm{~b}$ were diluted to $10 \mathrm{wt} \%$ in chloroform before the analysis. 
on the acid treatment of furan in the mixed methanol/water solvent.

\section{Conversion of furan in methanol/water}

Fig. 4a shows that the conversion of furan was very quick in methanol/water. This, however, was not accompanied with a proportional increase in benzofuran formation (Fig. 4b). Benzofuran yields barely exceeded $5 \%$. In addition, the abundance of the soluble polymer formed was also remarkably less in methanol/water than in methanol (Fig. 2a and c). Thus, the polymerization to the insoluble polymer was supposed to be the main reaction pathway for the conversion of furan in methanol/ water. The methanol/water volumetric ratio of $1: 1$ or $2: 1$ made no marked difference.

It is believed that water and methanol have very different solubility towards the soluble polymer. It can be found that in water, the abundance of the soluble polymer was also significantly less than that in methanol (Fig. 2a and b), but the amount of the insoluble polymer was significantly more ( $\mathrm{ca} .90 \%$ in water at $170{ }^{\circ} \mathrm{C}$ versus ca. $42 \%$ in methanol at $170{ }^{\circ} \mathrm{C}$ ). This result indicated that in water, the transformation of the soluble polymer into the insoluble polymer proceeded quickly, which was probably related to the low solubility of the polymer in water. In contrast, in methanol, a significant portion of the soluble polymer was retained in the reaction medium. Methanol probably helped to stabilize the soluble polymers.

The presence of water in methanol also affected the formation of intermediates such as 1,1,4,4-tetramethoxybutane. The abundance of 1,1,4,4-tetramethoxybutane was quite small in methanol/water (Fig. 4c), due to the equilibrium being shifted to the aldehyde side. The acetal was a relatively stable intermediate, formation of which created the chance and the time for the formation of benzofuran. It played essential roles in the suppression of the polymerization reactions. As was evidenced in methanol/water, without the significant formation of 1,1,4,4tetramethoxybutane, the polymerization dominated.

The conversion of furan over a higher loading of A70 was also investigated (Fig. 4). With the availability of more acidic sites, the conversion of furan (Fig. 4a) was relatively quicker. As mentioned above, 1,1,4,4-tetramethoxybutane (Fig. 4c) was the reaction intermediate. It was produced initially and soon it was further converted into benzofuran and dibenzofuran. Correspondingly, the formation of benzofuran and dibenzofuran (Fig. 4b and d), as well as the soluble polymer (Fig. S6 $\dagger$ ), was also promoted. This was understandable as the conversion of furan into benzofuran via the electrophilic substitution was catalyzed by the acid catalyst. Higher catalyst loading would accelerate the acid-catalyzed reactions. However, one drawback with the high A70 loading was that the formation of water via the etherification of methanol was accelerated (water content at the end was $24.7 \mathrm{wt} \%$ ), which consumed methanol and suppressed the formation of 1,1,4,4-tetramethoxybutane.

\section{Characterization of the insoluble polymer and the soluble polymer}

From the abovementioned results, it is concluded that the formation of polymer was the main reason for the low yield of benzofuran from furan, especially in water. To understand the
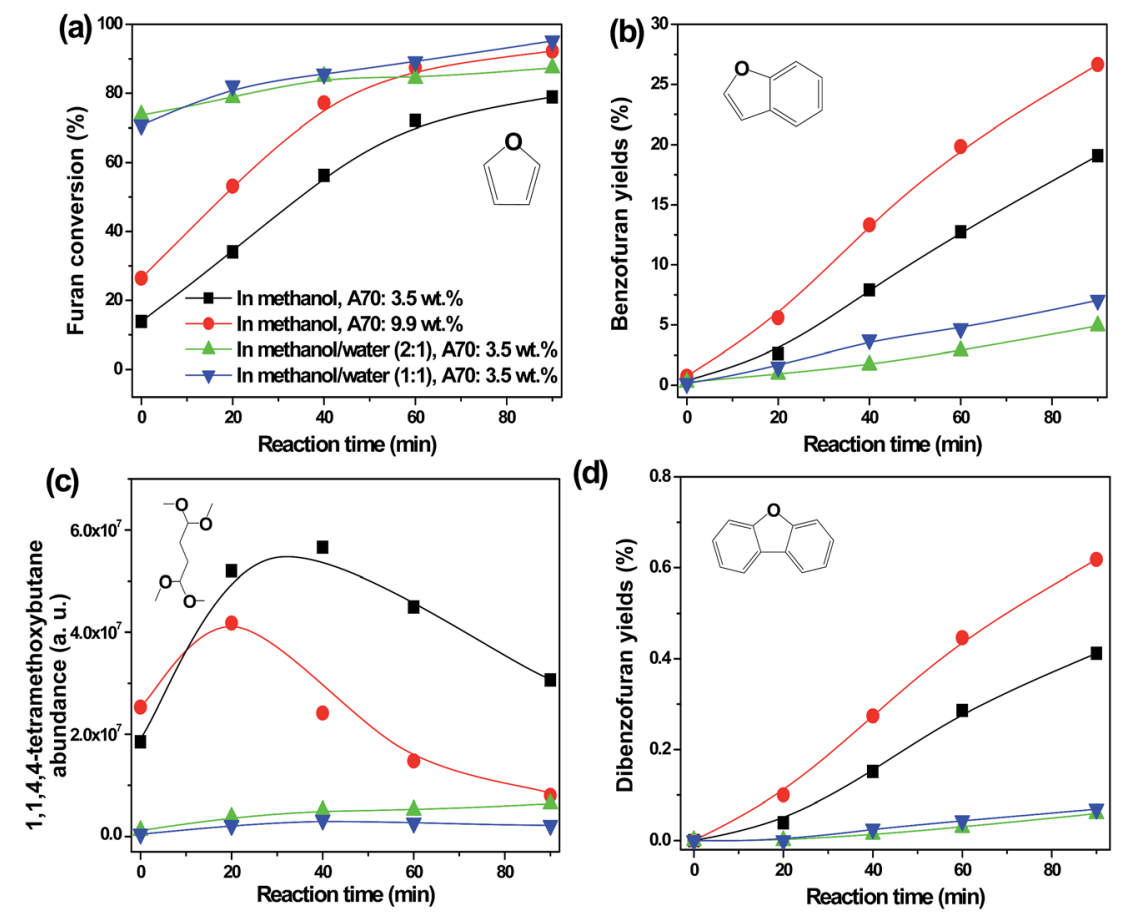

Fig. 4 The conversion of furan in methanol and in methanol/water. (a) The conversion of furan; (b) the yields of benzofuran; (c) the abundance of 1,1,4,4-tetramethoxybutane; (d) the yields of dibenzofuran. Reaction conditions: furan, $3 \mathrm{~g} ; \mathrm{T}=170{ }^{\circ} \mathrm{C}$; catalyst loading and solvent used are specified in Fig. 4a; stirring rate, 350 rpm; reaction time, $90 \mathrm{~min}$; pressure, autogenous vapor pressure. The ratio of methanol/water referred is the volumetric ratio. " $0 \mathrm{~min}$ " in the $x$-axis means the reaction temperature just reached the required temperature. 
intermediates involved in the polymerization reactions, the polymer formed in methanol (Run $5 \#$ and 6\#), in water (Run 1\#, $2 \#$ and $3 \#$ ) and in methanol/water (Run $4 \#$ ) with a different loading of A70 (Run $3 \#$ versus the others) and different reaction temperatures $\left(150\right.$ versus $\left.170{ }^{\circ} \mathrm{C}\right)$ were characterized. Fig. 5 shows the yields of the insoluble polymer and of benzofuran.

The results were in line with those in Fig. 1. In water, furan was mainly polymerized, with little formation of benzofuran. Interestingly, with a lower A70 loading (Run 3\#), there was less polymer formed and furan was not fully converted. The polymerization of furan mainly occurred inside the pores of the catalyst, which probably deactivates the catalyst. Deactivation of A70 due to polymerization was also observed in our previous study. ${ }^{23}$ After deactivation of the acid catalyst, further conversion of furan would be slowed down. Although the yield of the polymer in Run $3 \#$ was lower than that in Run $1 \#$ and $2 \#$, the selectivity for the polymer, which was defined by the amount of the polymer formed divided by the amount of furan converted, was close to $90 \%$. Obviously, with a lower or higher loading of the acid catalyst, the main reaction pathways of furan in water remained the same. Furan has a low solubility in water, which might also contribute to the polymer formation in Run $1 \#$, 2\# and $3 \#$. In Run $4 \#$ with the presence of $50 \%$ (by volume) of water, the polymerization was still significant, but the production of benzofuran was increased due to the presence of methanol (50\%). With methanol as the solvent, the formation of the insoluble polymer was suppressed to a significant extent.

Furan, with no other functionalities, is mainly polymerized in water in the presence of the acid catalyst. Understanding the functionality of the insoluble polymer will help to understand

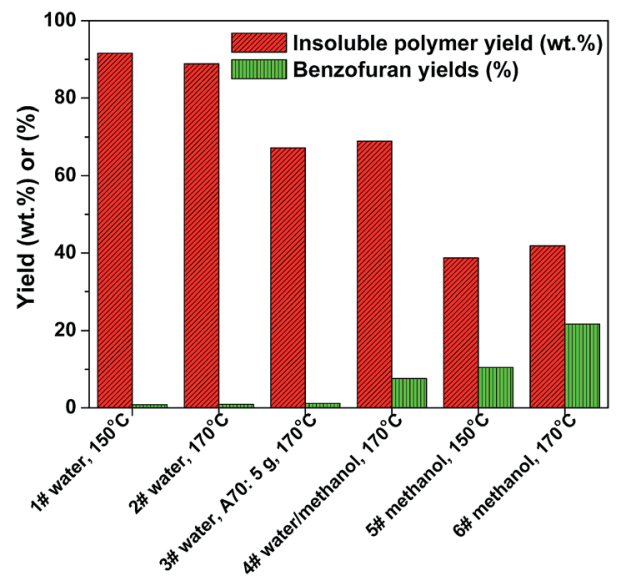

Fig. 5 Formation of insoluble polymer and benzofuran during the acid-treatment of furan. Typical reaction conditions: furan, $10 \mathrm{~g}$; water or methanol, $80 \mathrm{~mL}$; catalyst, Amberlyst 70 (A70); stirring rate, 350 rpm, reaction time, 90 min; pressure, autogenous vapor pressure. No sampling was performed during the experiments. Typical reaction conditions for individual experiments: $1 \#$ : water, $T=150^{\circ} \mathrm{C}$; $\mathrm{A70}, 10 \mathrm{~g}$. 2\#: water, $T=170{ }^{\circ} \mathrm{C}$; A70, $10 \mathrm{~g} .3 \#$ : water, $T=170{ }^{\circ} \mathrm{C}$; A70, 5 g. 4\#: water/methanol (volume/volume $=1: 1$ ), $T=170{ }^{\circ} \mathrm{C} ; \mathrm{A} 70,10 \mathrm{~g} .5 \mathrm{~A}$ : methanol, $T=150{ }^{\circ} \mathrm{C} ; \mathrm{A} 70,10 \mathrm{~g} .6 \#$ : methanol, $T=170{ }^{\circ} \mathrm{C} ; \mathrm{A} 70,10 \mathrm{~g}$ The conversion of furan in Run $1 \#, 2 \#$, and $4 \#$ was close to $100 \%$. In Run 3\# the conversion was ca. $75 \%$, while that in Run $5 \#$ and $6 \#$ were ca. $50 \%$ and $83 \%$, respectively.
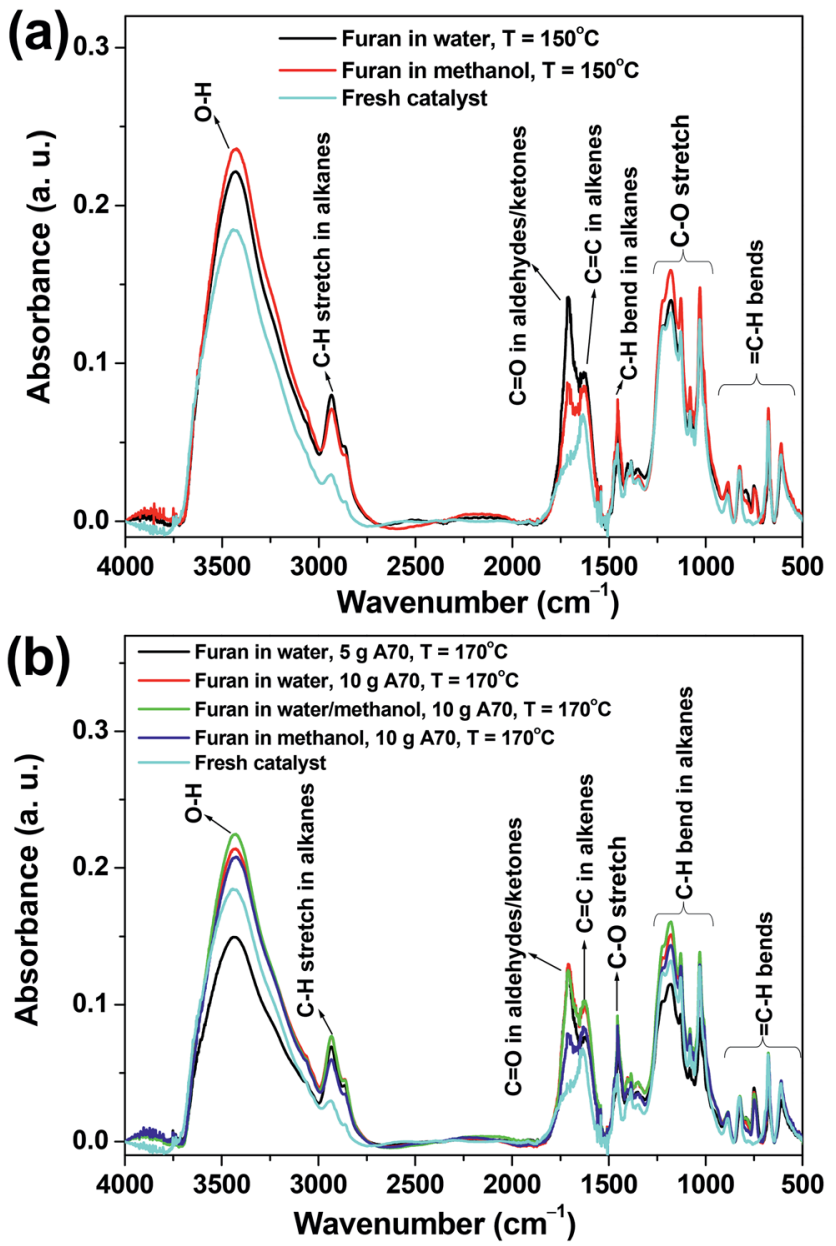

Fig. 6 FT-IR spectra of the fresh Amberlyst 7 and the used catalysts (polymer plus catalyst) obtained at (a) $150{ }^{\circ} \mathrm{C}$ and (b) $170{ }^{\circ} \mathrm{C}$. The typical reaction conditions for the catalysts experienced were described inside figures. The detailed conditions can refer to that specified in the caption of Fig. 5.

the reaction intermediates involved in the polymerization reactions. The insoluble polymer formed under the conditions specified in Fig. 5 was characterized with FT-IR, as shown in Fig. 6. The polymer shows a strong absorption for carbonyls, especially for the ones formed in water or in methanol/water. Evidently, the polymerization of furan involved the opening of the furan ring to form aldehydes or ketones. In fact, the formation of succinaldehyde has been detected with water as the solvent. This further confirms the important roles of aldehydes/ketones in the polymerization of furan. The absorption of the carbonyl group in the polymer formed in methanol was relatively weaker than that in the polymer formed in water. The aldehydes such as succinaldehyde could be converted into 1,1,4,4-tetramethoxybutane via acetalization, which protected these reactive functionalities and suppressed their involvement in the polymerization reactions.

Soluble polymer was the precursor for the formation of the insoluble polymer, characterization of which may also obtain important information about the reactive intermediates involved in the polymerization of furan. FT-IR characterizations 

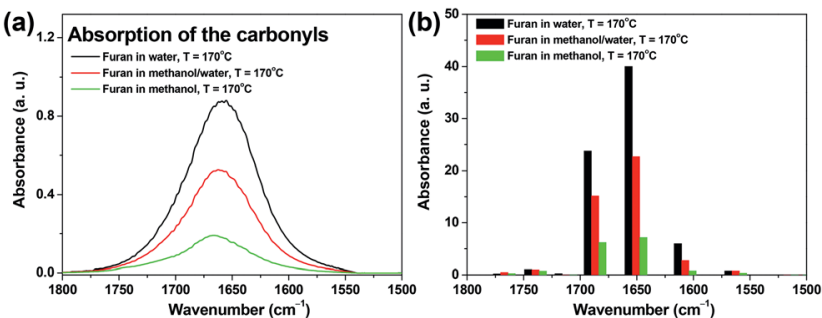

Fig. 7 (a) FT-IR spectra of the soluble polymers; (b) deconvolutions of the FT-IR spectra in Fig. 7a. The liquid products were diluted to 50\% (by volume) with methanol before the characterization with FT-IR.

of the soluble polymer in the liquid samples were carried out with the focus on the absorptions of carbonyls. Deconvolution of the spectra was also performed, as shown in Fig. 7. The absorption at $1696 \mathrm{~cm}^{-1}$ represents the $\mathrm{C}=\mathrm{O}$ stretching vibration of unsaturated aldehydes/ketones. The absorption at 1654 $\mathrm{cm}^{-1}$ represent the $\mathrm{C}=\mathrm{O}$ stretching vibration of the hydroxyl unsaturated aldehydes/ketones, whereas the absorption at 1606 $\mathrm{cm}^{-1}$ represents the aromatic $\mathrm{C}=\mathrm{C}$ ring breathing in aromatics with various types of substitution. Evidently, relatively more aldehydes/ketones and more aromatics with varied substitutions were observed in the polymer formed in water than in that formed in methanol. This result was in line with the characterization of the insoluble polymer with FT-IR (Fig. 6), wherein the polymer formed in water tended to have more aldehydes/ ketone groups on its surface. The presence of aromatics with fused-ring structures were also confirmed from the characterization of the soluble polymer with UV fluorescence, as shown in Fig. 8. The fluorescence spectra extended to $c a .425 \mathrm{~nm}$, indicating that the aromatics have 3 - to 5 -fused ring structures. Based on the above characterization, it was obvious that the polymerization of furan formed the polymer with aromatic ring structures and aldehyde/ketone functionalities.

Because polymer can be formed from furan in either methanol or water, further study on the difference of the polymer

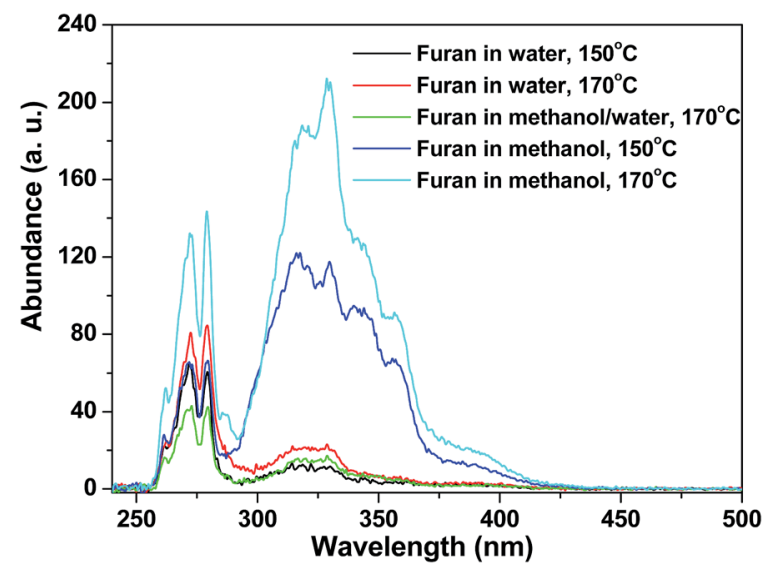

Fig. 8 Constant energy $\left(-2800 \mathrm{~cm}^{-1}\right)$ synchronous spectra for the soluble polymers in the liquid samples. The typical reaction conditions are described inside the figure. Detailed conditions are specified in the caption of Fig. 5.
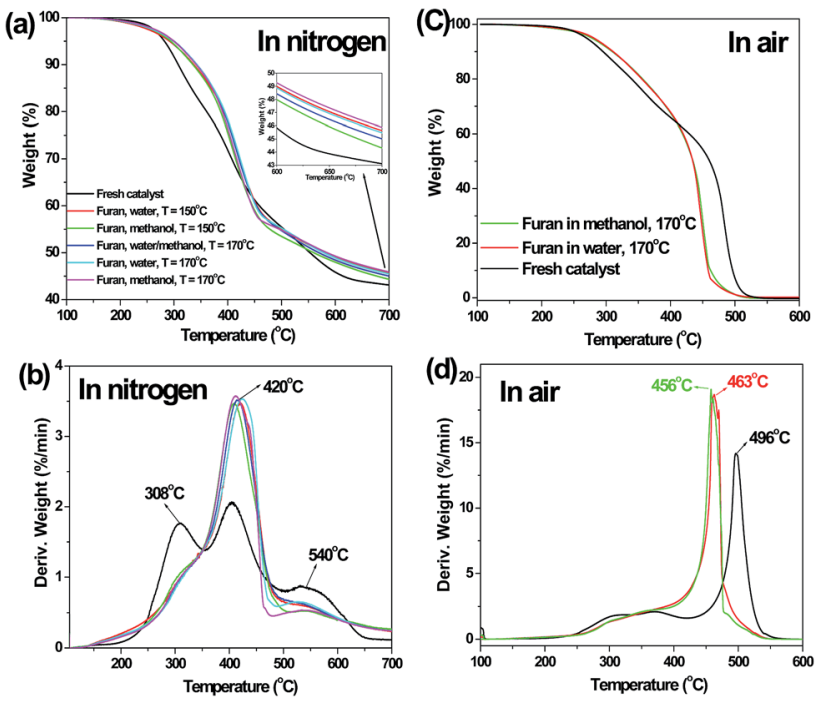

Fig. 9 TGA and DTG curves for the used catalyst (polymer plus catalyst) and fresh Amberlyst 70 catalyst. The fresh catalyst and the used catalysts were dried at $100{ }^{\circ} \mathrm{C}$ in nitrogen for $60 \mathrm{~min}$ before further heating to $700{ }^{\circ} \mathrm{C}$ in nitrogen and $600{ }^{\circ} \mathrm{C}$ in air at $10^{\circ} \mathrm{C} \mathrm{min}^{-1}$. The gas flow rate was $100 \mathrm{~mL} \mathrm{~min}^{-1}$. Typical reaction conditions experienced by the catalysts are described inside the figures. Detailed conditions are specified in the caption of Fig. 5.

produced in the two different solvents was conducted using TGA and elemental analysis. Pyrolysis and combustion behaviours of the polymer plus catalyst were performed with TGA in nitrogen and in air, respectively. As shown in Fig. 9, the weight loss was not significantly different for the fresh catalyst and for the polymer (plus catalyst) formed in water or in methanol. However, the formation of polymer inside the catalyst did change the pyrolysis behaviours of the catalyst and the used catalyst (polymer plus catalyst). The weight loss became more significant at ca. $420{ }^{\circ} \mathrm{C}$ for the used catalyst, while for the fresh catalyst the weight loss was also significant at 308 and $504{ }^{\circ} \mathrm{C}$. It was possible that the pyrolysis of the polymer mainly occurred at $c a .420{ }^{\circ} \mathrm{C}$. The combustion of the polymer (plus catalyst) formed in water or in methanol showed almost the exact same trends. From the TGA results, the differences in the polymer formed in water or in methanol in terms of pyrolysis or combustion behaviours were insignificant. The polymer was further characterized with an elemental analyzer to measure potential differences in their elemental composition.

Because the polymer was formed on/in the catalyst, the polymer plus catalyst was thus characterized as one entity. The carbon, hydrogen and oxygen contents in the polymer were calculated based on the mass ratio of the polymer to catalyst. As shown in Table 1, compared to that in the fresh catalyst, the content of oxygen and sulphur were decreased, while the content of carbon and hydrogen were increased correspondingly. The abundance of oxygen and sulphur were clearly diluted with the presence of the polymer on/in the catalyst as the polymer has no sulphur and lower oxygen content when compared with the catalyst. 
Table 1 Elemental composition of polymer plus catalyst (on a dry ashfree basis)

\begin{tabular}{|c|c|c|c|c|c|}
\hline \multirow[b]{2}{*}{ Entry } & \multirow[b]{2}{*}{ Experimental conditions } & \multicolumn{4}{|c|}{$\begin{array}{l}\text { Elemental } \\
\text { composition (wt\%) }\end{array}$} \\
\hline & & $\mathrm{C}$ & $\mathrm{H}$ & $\mathrm{S}$ & $\mathrm{O}$ \\
\hline 1 & Furan, water, A70: $5 \mathrm{~g}, T=170{ }^{\circ} \mathrm{C}$ & 69.5 & 4.7 & 2.8 & 23.0 \\
\hline 2 & Furan, water, A70: $10 \mathrm{~g}, T=150^{\circ} \mathrm{C}$ & 67.3 & 4.4 & 3.9 & 24.4 \\
\hline 3 & Furan, water, A70: $10 \mathrm{~g}, T=170^{\circ} \mathrm{C}$ & 66.6 & 4.2 & 3.9 & 25.3 \\
\hline 4 & $\begin{array}{l}\text { Furan, water/methanol, A70: } 10 \mathrm{~g}, T= \\
170^{\circ} \mathrm{C}\end{array}$ & 65.5 & 4.0 & 4.1 & 26.4 \\
\hline 5 & Furan, methanol, A70: $10 \mathrm{~g}, T=150^{\circ} \mathrm{C}$ & 63.5 & 3.6 & 5.7 & 27.2 \\
\hline 6 & Furan, methanol, A70: $10 \mathrm{~g}, T=170{ }^{\circ} \mathrm{C}$ & 63.4 & 3.9 & 5.2 & 27.5 \\
\hline 7 & Fresh Amberlyst 70 & 47.9 & 2.9 & 9.7 & 39.5 \\
\hline
\end{tabular}

Table 2 Elemental composition of the polymer (on a dry ash-free basis)

\begin{tabular}{|c|c|c|c|c|}
\hline \multirow[b]{2}{*}{ Entry } & \multirow[b]{2}{*}{ Experimental conditions } & \multicolumn{3}{|c|}{$\begin{array}{l}\text { Elemental } \\
\text { composition } \\
(\mathrm{wt} \%)\end{array}$} \\
\hline & & $\mathrm{C}$ & $\mathrm{H}$ & $\mathrm{O}$ \\
\hline 1 & Furan, water, A70: $5 \mathrm{~g}, T=170{ }^{\circ} \mathrm{C}$ & 78.2 & 5.4 & 16.4 \\
\hline 2 & Furan, water, A70: $10 \mathrm{~g}, T=150^{\circ} \mathrm{C}$ & 78.8 & 5.2 & 16.0 \\
\hline 3 & Furan, water, A70: $10 \mathrm{~g}, T=170^{\circ} \mathrm{C}$ & 77.9 & 5.1 & 17.0 \\
\hline 4 & Furan, water/methanol, A70: $10 \mathrm{~g}, T=170^{\circ} \mathrm{C}$ & 77.8 & 4.8 & 17.4 \\
\hline 5 & Furan, methanol, A70: $10 \mathrm{~g}, T=150{ }^{\circ} \mathrm{C}$ & 81.8 & 4.4 & 13.8 \\
\hline 6 & Furan, methanol, A70: $10 \mathrm{~g}, T=170^{\circ} \mathrm{C}$ & 80.1 & 4.9 & 15.0 \\
\hline 7 & Furan chemical & 70.6 & 5.9 & 23.5 \\
\hline
\end{tabular}

The carbon content of the polymer was in the range of $c a .78-$ $82 \%$, which is $c a .10 \%$ higher than that of furan (Table 2). Moreover, the contents of hydrogen and oxygen were lower than that in furan. Obviously, the formation of the polymer was accompanied by dehydration reactions. The dehydration was believed not to be significant; however, as the polymer formed in water still has relatively high oxygen content (ca. 17\% versus $23.5 \%$ in furan). The oxygen was possibly retained in the polymer in functionalities, such as aldehydes and ketones, or in the form of the ether bond connecting aromatic ring structures. The carbon content in the polymer formed in methanol was slightly higher than that formed in water and correspondingly the oxygen content was slightly lower. Methanol could transform the aldehyde functionality into acetals, which might be one reason for this slight difference. In addition, the reaction intermediates involved in the polymerization reactions were not the same in water as in methanol, which might also affect the elemental composition of the polymer.

\section{Conversion of furan in ethanol, 1-propanol and 2-propanol}

The conversions of furan in the other alcohol solvents (ethanol, 1-propanol and 2-propanol) were not as significant as that in methanol (Fig. 10). Benzofuran yields in the alcohols were also different (Fig. 10b), which is related to the different conversions of furan in the alcohols. However, the overall selectivities
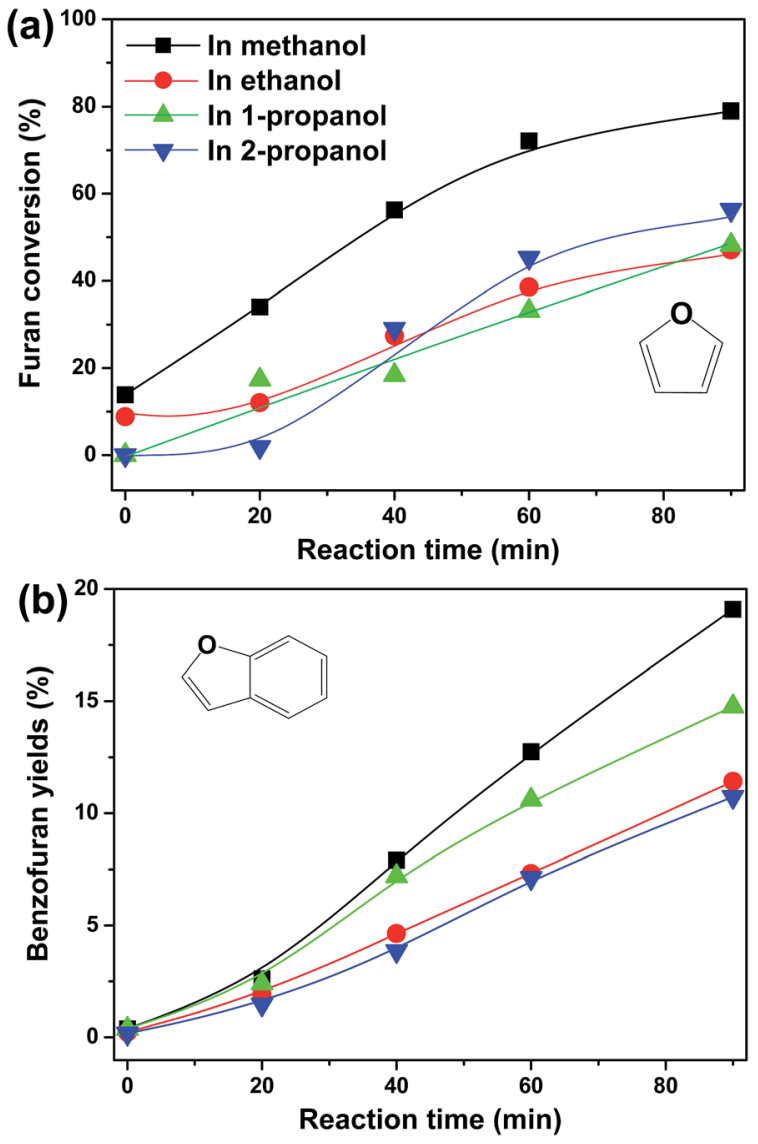

Fig. 10 The conversion of furan in the alcohols. (a) Furan conversion in the alcohols; (b) the yields of benzofuran in the alcohols. Reaction conditions: furan, $3 \mathrm{~g}$; solvent, $100 \mathrm{ml}$; catalyst, A70, $3 \mathrm{~g}$; stirring rate, 350 rpm; reaction time, $90 \mathrm{~min}$; pressure, autogenous vapor pressure. " $0 \mathrm{~min}$ " in the $x$-axis means the reaction temperature just reached the required temperature.

towards benzofuran, which eliminates the difference in furan conversion in the different alcohols, were not significantly different (ranging from 20 to $30 \%$ ). The alcohols investigated had similar capacities to suppress the polymerization reactions.

The soluble polymers formed in the various alcohols were not exactly the same (Fig. $2 \mathrm{~d}$ and S7 $\dagger$ ). The peaks at 317 and 330 $\mathrm{nm}$ reflected soluble polymers with different $\pi$-conjugated structures. The peak at $330 \mathrm{~nm}$ was more intensive in methanol than in other alcohols, indicating that methanol affected the formation of the soluble polymer in a way different from other alcohols, which was probably related to the smaller size of methanol.

\section{Conversions of furan in tetrahydrofuran (THF) and dimethyl sulfoxide (DMSO)}

The distinct effects of water and methanol on the polymerization of furan encouraged us to explore the conversion of furan in other solvents. Fig. 11 shows the conversion of furan in THF and in DMSO. THF is not an alcohol and is not involved much in the conversion of furan. It could not react with succinaldehyde to form the acetal to protect the reactive aldehyde 

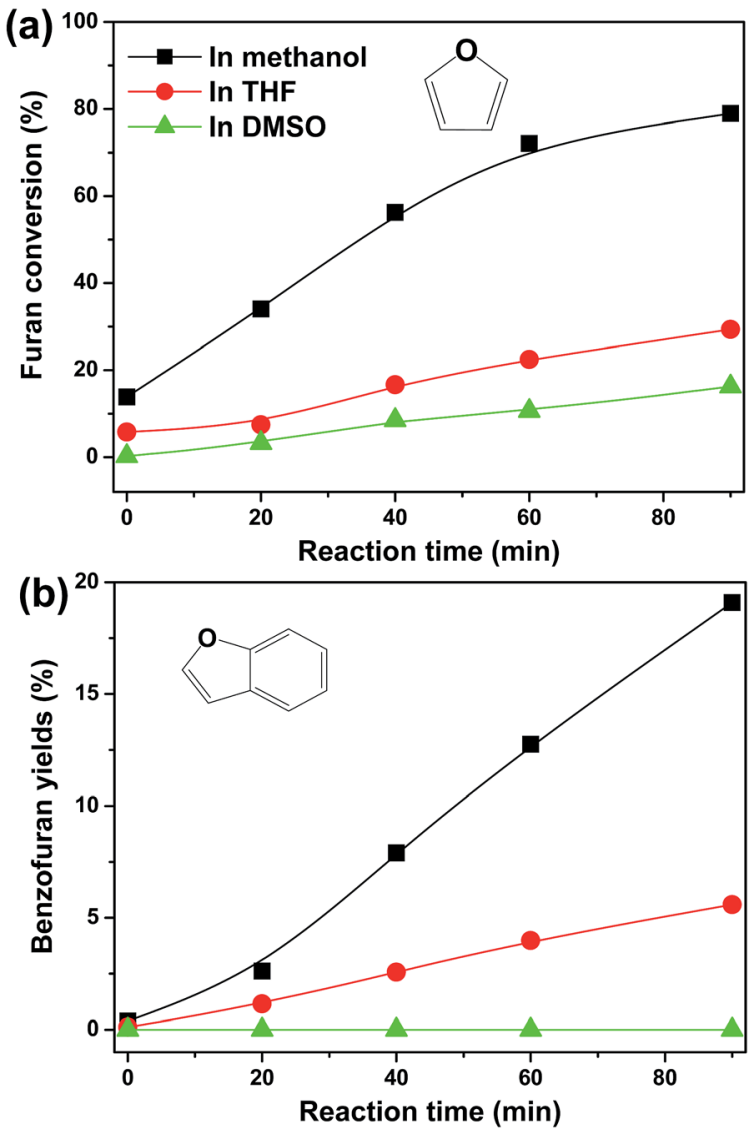

Fig. 11 The conversion of furan in THF and in DMSO. (a) Furan conversion in the solvents; (b) the yields of benzofuran in the solvents. Reaction conditions: furan, $3 \mathrm{~g}$; solvent, $100 \mathrm{ml}$; catalyst, A70, $3 \mathrm{~g}$ : stirring rate, $350 \mathrm{rpm}$; reaction time, $90 \mathrm{~min}$; pressure, autogenous vapor pressure. " $0 \mathrm{~min}$ " in the $x$-axis means the reaction temperature just reached the required temperature.

functionality. The yield of benzofuran barely exceeded $5 \%$ (Fig. 11b). THF was not an effective solvent for the conversion of furan into benzofuran.

DMSO was also not effective for the conversion of furan into benzofuran. The amount of benzofuran formed was negligible (Fig. 11b) and the conversion of furan was small (Fig. 11a). In addition, the soluble polymers formed from furan in DMSO

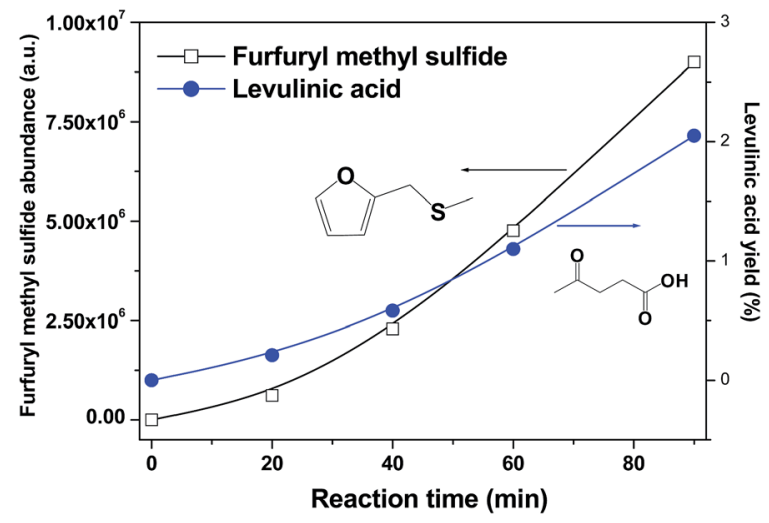

Fig. 12 The formation of levulinic acid and furfuryl methyl sulfide in acid treatment of furan in DMSO.

were also negligible (Fig. 2d). Evidently, the polymerization of furan was effectively suppressed in DMSO, which was similar to the suppression of the polymerization of HMF or furfural in DMSO. ${ }^{35,36}$ DMSO does not have a hydroxyl group as that in water or alcohols, and conversion of furan in DMSO was negligible. It seems that the hydroxyl group (either that in water or in alcohols) is very important for the conversion of furan via the opening of the furan ring.

In DMSO, instead of polymerization or conversion to benzofuran, furan was oxidized by DMSO to form $2(5 \mathrm{H})$-furanone or reacted with DMSO (or the derivative of DMSO), via electrophilic substitution, forming furfuryl methyl sulfide (Scheme 4, Fig. S8 and S9†). The detailed reaction route has not been understood yet. Furfuryl methyl sulfide might further react with furan, forming 2,2'-methylenebis-furan via electrophilic substitution. More interestingly, furfuryl methyl sulfide was also further converted into levulinic acid and methanethiol (Scheme 4). It is believed that some reducing agent(s) are involved in the transformation of furan in DMSO into furfuryl methyl sulfide. In our previous study on the conversion of xylose to furfural, ${ }^{10}$ we found that formic acid, the degraded product of furfural, was the hydrogen donor promoting the conversion of furfural into furfuryl alcohol. Formic acid was not detected in the conversion of furan in DMSO. Thus, some other reducing

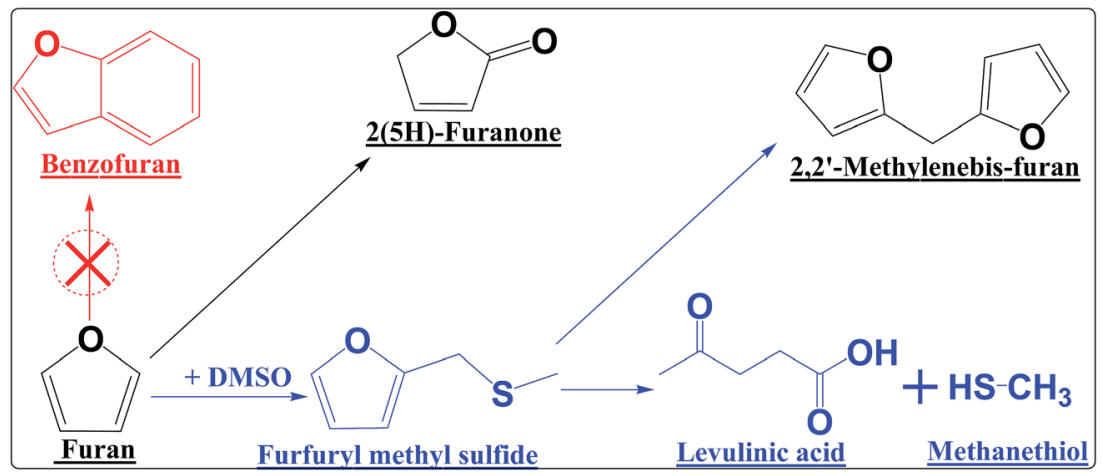

Scheme 4 A proposed reaction network for the conversion of furan in DMSO. All the compounds listed above were detected with GC-MS. 
agent must be involved in the reduction of DMSO, which is under current further investigation.

The conversion of furfuryl methyl sulfide into levulinic acid was very similar to the conversion of furfuryl alcohol into levulinic acid, ${ }^{37,38}$ due to the similar molecular configuration of the two furans. To the best of our knowledge, this is the first time for this reaction to be reported. The yields of levulinic acid and its precursor, furfuryl methyl sulfide, increased monotonically with reaction time (Fig. 12), which was a favorable reaction route. This result revealed the possibility for the production of levulinic acid from furan via modifying its molecular structures. The reaction conditions need to be further optimized to make furan the building block for chemical diversity and biofuels production, in addition to HMF and furfural, as another precursor for the production of levulinic acid.

\section{Conclusions}

Polymerization is the key challenge in the conversion of furan into benzofuran. Our results demonstrated that the polymerization of furan or its derivatives could be suppressed effectively using methanol as the solvent/reactant. The transformation of the aldehyde intermediate into the acetal with methanol was the key prerequisite to tackle the polymerization of furan. In water, without the conversion of succinaldehyde into the acetal, furan was mainly polymerized. In addition, the conversion of furan was sensitive to the molecular size of the alcohols. Methanol presented much higher capacity than higher alcohols such as ethanol and 1-propanol for the conversion of furan. Adjusting solvents could also modify the reaction pathways of furan during the acid-catalyzed conversion. In DMSO, instead of benzofuran, levulinic acid was produced, which diversifies the application of furan as a chemical feedstock. The conversion of furan in a wider range of solvents and acid catalysts needs to be explored to effectively upgrade the furan range of biofuels to the aromatic range of biofuels.

\section{Acknowledgements}

This study is supported by the Commonwealth of Australia under the Australia-China Science and Research Fund, as well as Curtin University of Technology through the Curtin Research Fellowship Scheme. This project received funding from ARENA as part of ARENA's Emerging Renewables Program.

\section{References}

1 D. Mohan, C. U. Pittman Jr and P. H. Steele, Energy Fuels, 2006, 20, 848-889.

2 X. Hu, C. Lievens, D. Mourant, Y. Wang, L. Wu, R. Gunawan, Y. Song and C.-Z. Li, Appl. Energy, 2013, 111, 94-103.

3 H. Wang, J. Male and Y. Wang, ACS Catal., 2013, 3, 10471070.

4 X. Hu, R. Gunawan, D. Mourant, C. Lievens, X. Li, S. Zhang, W. Chaiwat and C.-Z. Li, Fuel, 2012, 97, 512-522.

5 M.-J. Jeon, J.-K. Jeon, D. J. Suh, S. H. Park, Y. J. Sa, S. H. Joo and Y.-K. Park, Catal. Today, 2013, 204, 170-178.
6 M. S. Mettler, S. H. Mushrif, A. D. Paulsen, A. D. Javadekar, D. G. Vlachos and P. J. Dauenhauer, Energy Environ. Sci., 2012, 5, 5414-5424.

7 Q. Lu, W.-M. Xiong, W.-Z. Li, Q.-X. Guo and X.-F. Zhu, Bioresour. Technol., 2009, 100, 4871-4876.

8 M. J. Climent, A. Corma and S. Iborra, Green Chem., 2014, 16, 516-547.

9 D. M. Alonso, S. G. Wettstein, M. A. Mellmer, E. I. Gurbuz and J. A. Dumesic, Energy Environ. Sci., 2013, 6, 76-80.

10 X. Hu, C. Lievens and C.-Z. Li, ChemSusChem, 2012, 5, 14271434.

11 J. Guo, G. Xu, Z. Han, Y. Zhang, Y. Fu and Q. Guo, ACS Sustainable Chem. Eng., 2014, 2, 2259-2266.

12 X. Hu, Y. Song, L. Wu, M. Gholizadeh and C.-Z. Li, ACS Sustainable Chem. Eng., 2013, 1, 1593-1599.

13 X. Hu and C.-Z. Li, Green Chem., 2011, 13, 1676-1679.

14 A. A. Rosatella, S. P. Simeonov, R. F. M. Frade and C. A. M. Afonso, Green Chem., 2011, 13, 754-793.

15 T. Wang, M. W. Nolte and B. H. Shanks, Green Chem., 2014, 16, 548-572.

16 S. Eminov, J. D. E. T. Wilton-Ely and J. P. Hallett, ACS Sustainable Chem. Eng., 2014, 2, 978-981.

$17 \mathrm{X} . \mathrm{Hu}$, D. Mourant, Y. Wang, L. Wu, W. Chaiwat, R. Gunawan, M. Gholizadeh, C. Lievens, M. Garcia-Perez and C.-Z. Li, Fuel Process. Technol., 2013, 106, 569-576.

18 C. J. Gilbert, J. S. Espindola, W. C. Conner Jr, J. O. Trierweiler and G. W. Huber, ChemCatChem, 2014, 6, 2497-2500.

19 Y.-T. Cheng and G. W. Huber, ACS Catal., 2011, 1, 611-628.

20 S. Vaitheeswaran, S. K. Green, P. Dauenhauer and S. M. Auerbach, ACS Catal., 2013, 3, 2012-2019.

21 J.-L. Grandmaison, P. D. Chantal and S. C. Kaliaguine, Fuel, 1990, 69, 1058-1061.

22 X. Hu, C. Lievens, A. Larcher and C.-Z. Li, Bioresour. Technol., 2011, 102, 10104-10113.

23 X. Hu, R. J. M. Westerhof, L. Wu, D. Dong and C.-Z. Li, Green Chem., 2015, 17, 219-224.

24 X. Hu, S. Kadarwati, Y. Song and C.-Z. Li, RSC Adv., 2016, 6, 4647-4656.

$25 \mathrm{X} . \mathrm{Hu}, \mathrm{R}$. Westerhof, D. Dong, L. Wu and C.-Z. Li, ACS Sustainable Chem. Eng., 2014, 11, 2562-2575.

26 X. Hu, Y. Wang, D. Mourant, R. Gunawan, C. Lievens, W. Chaiwat, M. Gholizadeh, L. Wu, X. Li and C.-Z. Li, AIChE J., 2013, 59, 888-900.

27 B. Saha and M. M. Abu-Omar, Green Chem., 2014, 16, 24-38.

28 C. M. Cai, N. Nagane, R. Kumar and C. E. Wyman, Green Chem., 2014, 16, 3819-3829.

29 N. Shi, Q. Liu, T. Wang, L. Ma, Q. Zhang and Q. Zhang, ACS Sustainable Chem. Eng., 2014, 2, 637-642.

30 S. K. R. Patil and C. R. F. Lund, Energy Fuels, 2011, 25, 47454755.

31 X. Hu, L. Wu, Y. Wang, D. Mourant, C. Lievens, R. Gunawan and C.-Z. Li, Green Chem., 2012, 14, 3087-3098.

32 A. Corma, O. Torre and M. Renz, ChemSusChem, 2011, 4, 1574-1577.

33 A. Corma, O. Torre, M. Renz and N. Villandier, Angew. Chem., 2011, 123, 2423-2426. 
34 B. Faust, Modern Chemical Techniques, Royal Society of Chemistry, London: 1997, p. 70.

35 V. Choudhary, A. B. Pinar, S. I. Sandler, D. G. Vlachos and R. F. Lobo, ACS Catal., 2011, 1, 1724-1728.

36 A. S. Amarasekara, L. D. Williams and C. C. Ebede, Carbohydr. Res., 2008, 343, 3021-3024.
37 G. M. G. Maldonado, R. S. Assary, J. Dumesic and L. A. Curtiss, Energy Environ. Sci., 2012, 5, 6981-6989.

38 J.-P. Lange, W. D. Graaf and R. J. Haan, ChemSusChem, 2009, 2, 437-441. 